\title{
Interplay between mathematics and physics to catch the nature of a scientific breakthrough: The case of the blackbody
}

\author{
Laura Branchetti* \\ Department of Mathematical, Physical and Computer sciences, University of Parma, Parma 43124, Italy \\ Alessia Cattabriga $\odot^{\dagger}$ \\ Department of Mathematics, University of Bologna, Bologna 40126, Italy \\ Olivia Levrini ${ }^{\ddagger}$ \\ Department of Physics and Astronomy, University of Bologna, Bologna 40126, Italy
}

(Received 26 December 2018; published 23 September 2019)

\begin{abstract}
This paper aims to provide a contribution to the research in physics education regarding the interplay between mathematics and physics in teaching and learning physics at the university level. The argument is developed through a study focused on the historical case study of the blackbody that led Planck to make one of the most significant scientific breakthroughs in physics: the introduction of discreteness and quantization into physical processes. The study is methodologically guided by the model that Udhen, Karam, Pietrocola, and Pospiech elaborated to highlight the interplay between physics and mathematics within teaching and learning practices [O. Uhden, R. Karam, M. Pietrocola, and G. Pospiech, Modelling mathematical reasoning in physics education, Sci. Educ. Netherlands 21, 485 (2012).]. The model emphasizes the distinction between the technical and structural roles of mathematics in physics, with the latter role being argued to correspond to processes of mathematization and interpretation. We used this model to analyze Planck's original papers and to reconstruct the reasoning that, thanks to the structural role played by mathematics, paved the way for the quantistic scientific breakthrough. The results of the analysis led us to design a teaching tutorial that we implemented with mathematics and physics university students. Students' reactions are reported to discuss the educational potential of the approach beyond the specific case and to argue for its potential general application to other similar physics topics.
\end{abstract}

DOI: 10.1103/PhysRevPhysEducRes.15.020130

\section{INTRODUCTION}

At any level, instructional materials tend to pay little, if any, attention to fleshing out the authentic roles that mathematics plays in the development of new ideas in physics. In this paper, we argue that if we wish to support students in understanding the structural roles of mathematics in the process of knowledge construction in physics, specific research-based approaches to design teaching materials have to be developed, also in collaboration with the research community in mathematics education. The approach we discuss here uses the framework elaborated by Uhden, Karam, Pietrocola and Pospiech [1] on the interplay

\footnotetext{
*laura.branchetti@unipr.it

†alessia.cattabriga@unibo.it

olivia.levrini2@unibo.it
}

Published by the American Physical Society under the terms of the Creative Commons Attribution 4.0 International license. Further distribution of this work must maintain attribution to the author(s) and the published article's title, journal citation, and DOI. between mathematics and physics. The framework is applied to analyze the case of blackbody and to build research-based instructional materials on this topic. In the next section, we argue why we chose the blackbody case and how the approach could be a reference for analyzing additional similar cases.

\section{A. The case of blackbody and beyond}

The redefinition of several physical base units planned for 2019 will attach a new conceptual and epistemological status to physical constants, as they will become the pillars of physical measurements [2].

Among the seven defining constants that will represent the basis of the new Système International d'Unités (SI), two were introduced by Planck in 1900: $h$ and $k$, the Planck constant and Boltzmann constant, respectively.

The introduction of $h$ and $k$ was part of a wider research program to find the blackbody distribution law, bridging gas theory and radiation theory through a pure thermodynamic approach [3]. Planck's approach attached explicit relevance to the search for universal constants. It was indeed coherent 
with a special epistemological view where absolute quantities were the pillar of a general, universal, objective, necessary and perennial knowledge [4]:

"It is not we who create the outside world because it is convenient, but the outside world which imposes itself on us with a primal violence. We must insist on this in our positivism-impregnated age. When in the study of any natural phenomenon we move from that which is particular, conventional and causal to that which is general, objective and necessary, all we do is look for the independent behind the dependent, the absolute behind the relative, the perennial behind the transitory." [5] (p. 157).

Within this research program and in concordance with this epistemological orientation, Planck triggered one of the most significant scientific breakthroughs, which led discreteness and quantization to question the basic assumption of the continuity of physical processes [3,6,7]. The day of Planck's presentation of the paper Zur Theorie des Gesetzes der Energieverteilung im Normalspektrum (On the Theory of the Law of Energy Distribution in Normal Spectrum) (see Ref. [8]) to the German Physical Society-December 14, 1900-is conventionally considered the birthday of quantum physics.

The case of the blackbody has also been widely studied from a historiographical point of view because it appears to challenge the Kuhnian model of the scientific revolution, which presumes the revolutions are caused by a change of paradigm, followed by changes in models, procedures, and principles $[3,9]$. In this case, the change in mathematical models anticipated their interpretation and the formulation of a new paradigm. This thesis is supported, for example, by Drago [10], who focused his analysis on the special role played by mathematics.

In spite of the foundational role of Planck's constant in physics and the historical relevance of the blackbody case, this topic is allotted relatively little space in physics education, maybe because of its deep complexity. Around the world, a sort of pseudohistorical standard narrative has been consolidated, also at the university level. As Dougal [11] (p. 438) states, "it is likely that the authors of the incorrect (or, more often, remarkably vague) accounts have attempted to keep to a minimum the complication of the historical development of the study of blackbody radiation during the four years leading up to 1900 when the Planck postulate $E=h \nu$ was first published [8]. Many authors have been too selective in their choices of what theoretical material to include and, what is worse, they have excluded from their accounts all but the most general references to the results of appropriate experiments."

The narrative usually aims to acknowledge Planck as one of the fathers (if not the father) of quantum physics and/or to ascribe a central position to the blackbody problem.
Consistently with these aims, the style chosen by the textbooks is mainly informative (as opposed to argumentative). A prototypical sentence is "Introducing $h$, Planck showed that energy emitted by a blackbody was divided into quanta" it does not contain any reference to the process that led Planck to his results but merely weaves a historical storytelling to frame them.

University textbooks usually tackle the topic in much more detail but, even here, Planck's argumentation is seldom tracked. A well-written and compelling example comes from Stanford University online materials: The origins of quantum theory, by Cathryn Carson [12]. The paragraph "Introducing $h$ " is the following:

"The story began inconspicuously enough on December 14, 1900. Max Planck was giving a talk to the German Physical Society on the continuous spectrum of the frequencies of light emitted by an ideal heated body. Some two months earlier this 42-year-old theorist had presented a formula capturing some new experimental results. Now, with leisure to think and more time at his disposal, he sought to provide a physical justification for his formula. Planck pictured a piece of matter, idealizing it somewhat, as equivalent to a collection of oscillating electric charges. He then imagined distributing its energy in discrete chunks proportional to the frequencies of oscillation. The constant of proportionality he chose to call $h$; we would now write $E=h \nu$. The frequencies of oscillation determined the frequencies of the emitted light. A twisted chain of reasoning then reproduced Planck's postulated formula, which now involved the same natural constant $h$. Looking back on the event, we might expect revolutionary fanfare. But as so often in history, matters were more ambiguous. Planck did not call his energy elements quanta and was not inclined to stress their discreteness, which made little sense in any familiar terms. So the meaning of his procedure only gradually became apparent. Although the problem he was treating was pivotal in its day, its implications were at first thought to be confined."

The text is rich in precise details that nicely frame Planck's contribution within the history of science. Yet these details still do not allow a reader to grasp and reconstruct Planck's reasoning. Another story that is very frequent in textbooks is the following: Planck is said to have introduced $h$ to solve the problem of ultraviolet catastrophe and to match the Wien and the Rayleigh-Jeans laws, which were valid at different points of the spectrum; Walker's textbook [13] for high school and the manual by Halliday, Resnick, and Krane for university [14] use similar strategies. This interpretation is historically inaccurate (the work of RayleighJeans is a reaction to Planck's hypothesis) [3], but what is more interesting to us is that it neglects the real problem that Planck did address. 
These examples show that the texts are usually characterized by segments of information which form a chronological narrative of what Planck achieved. The texts do not contain those inferential connections that are needed to shade light on how Planck's reasoning led to a scientific breakthrough.

The study presented in this paper aims to contribute to filling this lack and suggests a way to analyze the original articles that Planck published in 1900 and 1901 [8,15,16], in order to flesh out the nature and mechanism of the scientific breakthrough therein.

Still more than this, the paper aims to show how this case can provide a significant opportunity to deal with the important educational issue we mentioned in the beginning of the introduction: students' difficulties to understand the real role of mathematics in the development of new ideas in physics. Because of this focus, the study has an explicit educational orientation. Still because of this focus, the study does not intend to be an original contribution to the historical debate but simply refers to the history of physics as a tool for teaching and learning. Although based on a case study, the paper has the ambition to contribute to the wider debate in physics education research about the role played by mathematics in physics teaching and learning, and the main research questions go beyond the specific case: What instructional materials, curricular choices, and activities can support university teaching to boost students to understand the authentic roles of mathematics in the development of new ideas in physics? In particular, we articulate the above question into two subquestions: What instructional materials and activities can support university teaching to highlight the structural role of mathematics in the development of new ideas in physics, and overcome the tendency to reduce it to a mere technical instrument $[1,17-$ 20]? What is the effect of these materials and activities on students' understanding, epistemologies, and skills development? In the paper we address explicitly the first question by proposing a tutorial that focuses on the different roles played by mathematics in the blackbody case. Moreover, we pave the way to deal with the second one reporting about a pilot study where we collected some reactions to the materials from university students. The discussion on such reactions is the basis for further studies, specifically aimed to investigate the effects of the proposed activities on students' learning.

\section{B. Paper structure}

The paper is articulated as follows: in the next section (Sec. II), we present the theoretical framework about the interdisciplinarity between mathematics and physics that oriented the study; we then present the lens and methods we used for analyzing Planck's original papers and the results of our analysis (Sec. III). Starting from the results of the analysis, we prepared a tutorial document (reported in the Appendix) for university students where Planck's reasoning is reconstructed so as to highlight the interplay between mathematics and physics that characterizes it: in Sec. IV we describe how we used the tutorial with university students who participated in the activity. Students' reactions will allow us to comment in the final discussion (Sec. V) about the educational value of our interdisciplinary reconstruction of a scientific breakthrough, also beyond the specific case of blackbody. Final remarks on the generalizability of the approach will close the paper.

\section{THEORETICAL FRAMEWORK: INTERPLAY BETWEEN MATHEMATICS AND PHYSICS AND INTERDISCIPLINARITY}

The interplay between mathematics and physics is the focus of some interesting research. Many of these studies aim to address the problem expressed by Karam in the introduction to the special issue of the journal Science and Education: "in physics education, it is usual to find mathematics being seen as a mere tool to describe and calculate, whereas in mathematics education, physics is commonly viewed as a possible context for the application of mathematical concepts that were previously defined abstractly. [...] the analysis of historical case studies frequently broadens our understanding of this interplay [between mathematics and physics]" [18] (p. 487).

In a recent paper, Tzanakis [21] discusses a comprehensive approach called history-pedagogy-mathematics/ physics (HPM/Ph) that he has been developing in order to stress the relevance of the interplay of the two disciplines as the essence of their authentic historical evolution.

To support such a thesis, he discusses examples of historical cases, such as "(a) measuring the distance of inaccessible objects; (b) rotations, space-time, and special relativity; (c) differential equations, functional analysis, and quantum mechanics" [21] (p. 1). All these examples illustrate the basic assumptions of the HPM/Ph approach concerning the "historicity" of both mathematics and physics and their co-evolution, in an interwoven and bidirectional way. On the one hand, orienting from mathematics to physics, "mathematics is the language of physics, not only as a tool for expressing, handling and developing logically physical concepts, methods and theories, but also as an indispensable, formative characteristic that shapes them, by deepening, sharpening, and extending their meaning, or even endowing them with meaning" [21] (p. 6). On the other hand, orienting from physics to mathematics, "physics constitutes a (or maybe, the) natural framework for testing, applying and elaborating mathematical theories, methods and concepts, or even motivating, stimulating, instigating and creating all kinds of mathematical innovations" [21] (p. 6).

Further examples of the role of mathematics in physics are presented in papers of the special issue on Science and Education (2015): according to Brush, for instance, mathematics is an instigator of scientific revolutions [22]; Kragh 
stresses the creative power of formal analogies in physics, showing that mathematics provides formal structures that enable us to reason about new phenomena, starting from known ones [18].

Moving from a historical-epistemological perspective on the interplay between mathematics and physics to an educational view, a fundamental contribution has been given to physics education by Tuminaro and Redish [23] who proposed and elaborated the perspective of the epistemic game by adapting and enriching the definition introduced earlier by Collins and Ferguson in Ref. [24]. Tuminaro and Redish relied on the definition of the epistemic game as "a coherent activity that uses particular kinds of knowledge and processes associated with that knowledge to create knowledge or solve a problem" [25] (p. 24).

The researchers used the notion of epistemic game to describe students' attitudes to problem solving in terms of locally coherent goal-oriented activities (the epistemic games). These provide information on students' tacit expectations about how to approach physics problems and in particular about their understanding and use of mathematics.

Among the possible approaches to problem solving in physics, analyzing experts' and students' strategies, Tuminaro and Redish have identified six epistemic games (in order of most to least intellectually complex): (i) mapping meaning to mathematics; (ii) mapping mathematics to meaning; (iii) physical mechanism game; (iv) pictorial analysis; (v) recursive plug and chug; (vi) transliteration to mathematics.

Mapping meaning to mathematics is the most intellectually complex. Students who play this game begin by forming a conceptual understanding of the physical situation described in the problem statement and then progress to a quantitative solution. The knowledge base for this game consists of a set of physics and mathematics resources: these can be fundamental physics principles (e.g., Newton's laws or the electromagnetic induction law), intuitive mathematics knowledge, symbolic forms, but also reasoning primitives [25] such as "agent causes effect," which are abstractions from diSessa's phenomenological primitives [26].

When playing mapping mathematics to meaning the students are observed developing a conceptual story corresponding to a particular physics equation. The ontological components are the same as those in the previous game. The difference between the two is in their structure: while in mapping meaning to mathematics students translate a conceptual story into mathematical expressions, here the starting point is a physics equation that is used as the basis in developing a conceptual story.

Their empirical studies show that university teaching seems to fail to develop the knowledge, resources, and competences needed to activate those complex games while tending, on the contrary, to encourage the least intellectually complex games. Among these, the recursive plug-and-chug game is very frequent and is recognized when students plug quantities into physics equations and produce numeric answers, without any conceptual understanding of the physical implications of their calculations. The only element of the knowledge base for this game is the intuitive syntactic (not conceptual) understanding of physics symbols: intuitive mathematics knowledge, reasoning primitives, symbolic forms, and interpretive devices are not put into play here. Another game that reveals a possible weak link between mathematical procedures and the description of physical phenomena is the physical mechanism: students try to construct a physically coherent and descriptive story based on their intuitive sense of physical mechanism, using only reasoning primitives without any explicit reference to physics principles or equations. It is closer to more sophisticated games than the recursive plug and chug, but not enough to allow the students to effectively tackle nontrivial physical problems.

Another well-known model within the PER community is the model developed by Uhden, Karam, Pietrocola, and Pospiech [1]. Its functions are not specifically defined by the authors who instead write:

"It [the model] is intended to serve as a guiding framework when facing aspects related to mathematical reasoning in physics education.

This pertains to many aspects: lecturers explaining physical concepts, teachers designing classroom practice, researchers (and also teachers) inventing new tasks or diagnosing students' reasoning processes, just to mention some possible occasions. What is common for all of them is the need to have an underlying conceptual position towards the role of mathematics in physics education" [1] (p. 499).

The model, schematized in Fig. 1, is a revised version of the modeling cycle of Blum and Leiß [27]: the main changes concern the explicit choice to exploit (as core of the model) the entanglement of mathematics and

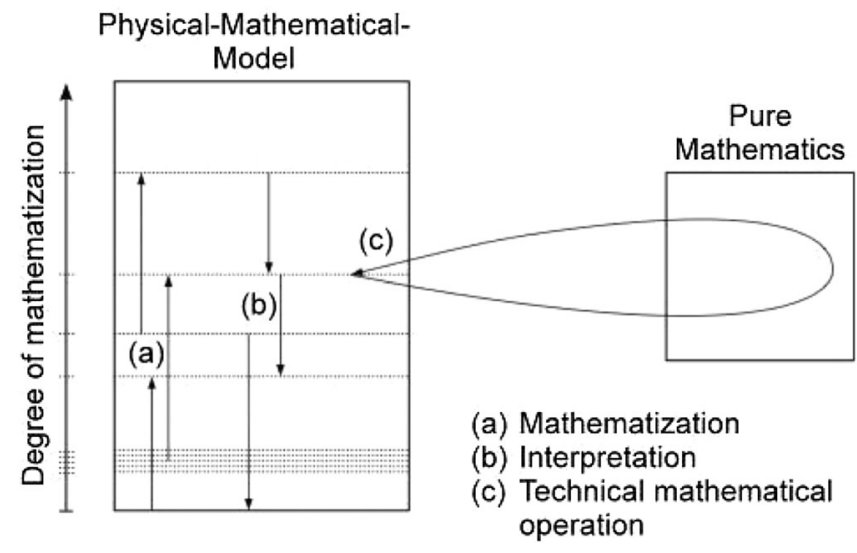

FIG. 1. Model of the use of mathematics in physics elaborated by Uhden, Karam, Pietrocola, and Pospiech in Ref. [1] (p. 497). 
physics, as well as the distinction between the technical and structural roles played by mathematics in physics, and between the different types of skills that are needed to acknowledge and manage the two different roles (the distinction between structural and technical roles of mathematics in physics was already stressed by Pietrocola and Pospiech in previous papers [28,29]).

While the structural skills are inseparable from physics, the technical skills can also exist independently of other disciplines. In their words: "the technical skills are associated with pure mathematical manipulations whereas the structural skills are related to the capacity of employing mathematical knowledge for structuring physical situations." [1] (p. 493).

In the figure representing the model, the technical skills are represented by the loop that moves from the rectangle and back (c). They do not have any physical reference per se: they are purely mathematical abilities "related to the instrumental domain of algorithmic rules (e.g., isolating a variable, operating with fractions, differentiating or integrating a function, and solving an equation), to the straightforward consult of a relation in a given list (e.g., differentiation rules, trigonometric identities, and moments of inertia) or to the quotation of properties and theorems using arguments of authority (e.g., Pythagoras' or Stokes' theorem and the associative property)" [1] (p. 498).

The structural skills correspond to the processes called (a) mathematization and (b) interpretation and are represented within the rectangle to show the essential interweaving of mathematics and physics.

Mathematization concerns the process of turning a physical problem into mathematics (at different degrees), while interpretation is "related to the ability of 'reading' equations, stating their meaning with the use of words and schemes, identifying special or limiting cases and making physical predictions from the formalism" [1] (p. 498).

The two processes of mathematization and interpretation strongly recall the epistemic games of, respectively, mapping meaning to mathematics and mapping mathematics to meaning, but the model does not refer specifically to problem solving activities and does not aim to describe the inner mechanism that students activate to address a physical problem.

As we will describe in more detail in the next section, we chose the model of Udhen and colleagues as a main reference to analyze the original papers of Planck and flesh out the interplay between mathematics and physics that structures Planck's reasoning. However, our objective is to use our analysis as a basis to build teaching materials designed to support and enable university students to understand and reflect on the role of mathematics in the process of knowledge construction in physics.

In particular, our analysis of Planck's papers will show how the blackbody can be used as an example to exploit all the roles of mathematics in physics that the previous review highlighted:
(1) instrumental or application: mathematics as a tool for physics, and physics as a context of application for mathematics [1];

(2) mathematics as instigator of scientific revolutions [22]: a novelty in a scientific field can be "dragged" by a formal improvement, a mathematical conjecture, a derivation not supported in the beginning by physical evidences;

(3) mathematics as source of creative power in physics [30]: mathematics can provide physics with unique reasoning strategies, like formal analogies, that lead physics to evolve through the exploitation of formal expressions and manipulations;

(4) mathematics as structural in physical modeling: mathematization and interpretation are crucial in physical modeling [1] and in problem solving [23]. In the paper, we will use, as well as the word interplay, the more demanding word interdisciplinarity between mathematics and physics. In this use, we rely on Thompson's definition as presented in Ref. [31] (p. 16). We speak of interdisciplinarity when disciplines mutually integrate, interact, and blend; we speak of multidisciplinarity when disciplines are juxtaposed, sequential, and coordinating. With respect to the previous list that sums up the possible ways in which mathematics and physics can interplay, we will speak of interdisciplinarity between mathematics and physics in the last three cases where mathematics and physics induce, respectively, relevant changes in the other discipline's epistemology, constraints, and methods. When mathematics is purely instrumental and physics a mere context of application, we can speak of multidisciplinarity.

\section{THE M-I-T ANALYSIS OF PLANCK'S REASONING}

As already anticipated, the analysis will focus on three of Planck's papers:

- On an Improvement of Wien's Equation for the Spectrum [15];

- The Theory of the Energy Distribution Law of the Normal Spectrum [8];

- On the Law of the Energy Distribution in the Normal Spectrum [16].

The first includes the mathematical conjecture that allowed Planck to draft his version of the distribution law for the spectral density and, as a result, provides a fruitful example of mathematization (see Ref. [1]). The second is the famous paper that Planck presented at the German Physical Society on December 14 to explain his theoretical interpretation [1] of the distribution law. In the third paper he proposed a more aware summary of the crucial steps he had taken "to find an alterable link in the chain of reasons resulting in the Wien's energy distribution law [and] to remove this link from the chain and create a suitable substitute" [16] (p. 553). Here Planck rigorously and theoretically derives the laws presented in the previous papers, with more details 
on the technical passages and their implications on the interpretation, coming to the values of the universal constants $h$ and $k$ and to the relation $E=h \nu$. The papers have been analyzed by applying the [1] scheme as a lens to highlight the crucial steps of the mathematization and interpretation processes, as well as the technical operations. The analysis has been carried out so as to exploit both the macro phases of Planck's reasoning and the critical details that led to the scientific breakthrough.

The macro phases are as follows:

(1) mathematical (formal) improvement of Wien's law with an expression of the spectral density that could fit better with the empirical data;

(2) construction of a model in analogy with Boltzmann's approach to thermodynamics and derivation of the law within a physical theory, as well as the derivation of the values of the universal constants $h$ and $k$ and the relation between energy and frequency. The first phase corresponds to the first article, and the second phase to the last two articles.

For each phase, Planck's reasoning is reconstructed in logical steps related to mathematization (M), interpretation (I) or mathematical technics (T). After the M-I-T detailed analysis, we sum up the general features of Planck's reasoning with regard to the interplay between mathematics and physics, both phase by phase, and as an overall synthesis (Sec. III C).

Ideally, the reader could follow the analysis of Planck's papers by contextually reading the original texts. However, the reconstructions of Planck's reasoning have been carried out and here reported according to three criteria of (a) faithfulness to the original text, (b) completeness (it is built so as to include all the details that a reader may need to follow the reconstruction of Planck's reasoning), (c) effectiveness to flesh out the critical details needed to highlight the interplay between mathematics and physics.

Each M-I-T analysis of Planck's reasoning is anticipated by a subsection describing the fundamental question and the general scientific and historical setting that preceded Planck's paper. The scope of these subsections is to contextualize Planck's reasoning so as to better understand it.

\section{A. Phase 1-Mathematical (formal) improvement of Wien's law}

\section{Before Planck}

The title of the first paper of Planck we consider here is in itself very significant: On an Improvement of Wien's Equation for the Spectrum [15]. The version of Wien's equation that Planck aimed to improve looks like the following:

$$
u(\nu, T)=\frac{8 \pi \nu^{3}}{c^{3}} a e^{-b \nu / T},
$$

with $a, b>0$ (note that this expression of Wien's law is not the equation that Wien wrote in 1896, but a formulation that
Planck wrote in 1899, after considering the energy of a Hertzian resonator and of the energy and entropy of the radiation field [32] [p. 46]). The equation expresses the distribution over the frequencies of the energy density (the spectral density) emitted by a blackbody at thermal equilibrium, i.e., at a given, constant temperature $T$.

The equation was built on Kirchhoff's definition of a blackbody as a perfect absorber, then as a "model of models" of emitters, depending solely on temperature and independent of the geometrical shape or material. Kirchhoff had defined a blackbody in 1860 as "the supposition that bodies can be imagined which, for infinitely small thicknesses, completely absorb all incident rays, and neither reflect nor transmit any. I shall call such bodies perfectly black, or, more briefly, black bodies" [33] (p. 276). Kirchhoff had already shown in 1859 that "the ratio of power of emission to the power of absorption, common to all bodies, is a function depending on the wavelength (of the radiation emitted or absorbed) and the temperature" [34] (p. 726). Thus, a body with power of emission equal to $1(e=1)$ - a perfect absorber-in thermal equilibrium would have emitted a universal spectrum, i.e., a spectrum that did not depend on the shape and on the material of the body, but only on its temperature. In order to produce "black radiation" in lab conditions, Kirchhoff created a cavity with a pinhole suitable for absorbing all incidental rays. The radiation emitted from the pinhole (Fig. 2) has a continuous spectrum (Fig. 3). Twenty-five years after the first law of Kirchhoff, in 1884, Boltzmann had given a theoretical derivation of a law obtained empirically by Stefan in 1879-that the total energy emitted per unit surface and unit time by a heated body is proportional to the fourth power of its absolute temperature:

$$
I=\sigma T^{4},
$$

where $\sigma$ is a constant of proportionality.

At the end of the XIX century, an open problem was to find an expression of the spectral density in order to mathematically describe the spectrum of the blackbody

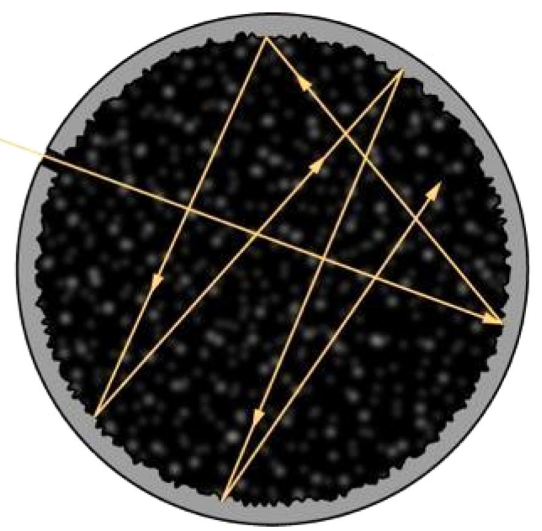

FIG. 2. The blackbody as a cavity (image taken from Ref. [35]). 


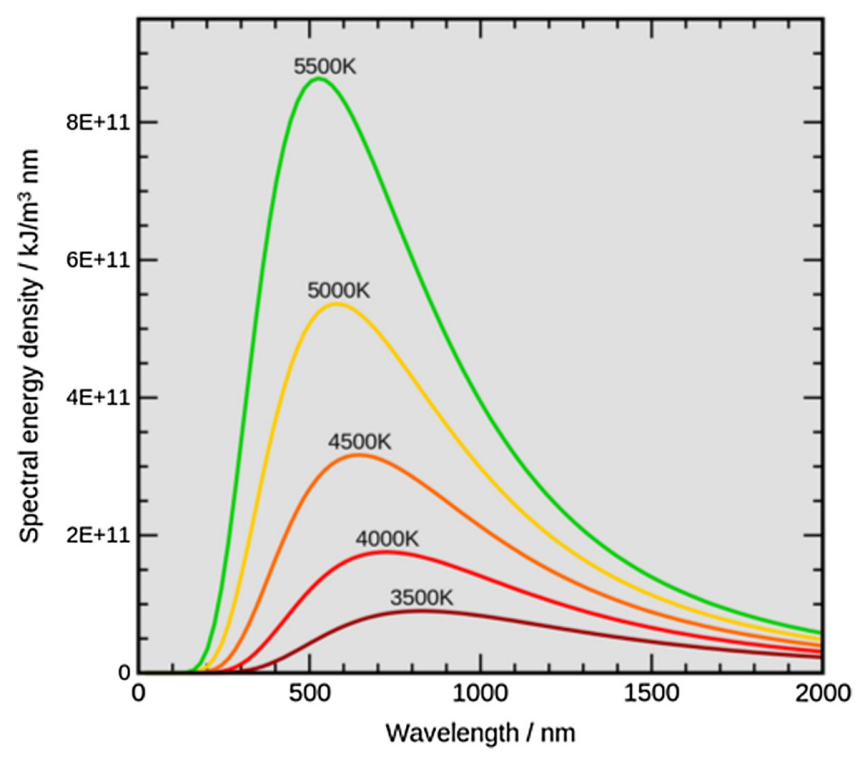

FIG. 3. Blackbody radiation as a function of wavelength for various absolute temperatures (image taken from Ref. [36]).

emitted radiation and its dependence on temperature (see Fig. 3). There were at least three reasons: the first, more empirical, was to find the mathematical expression that could fit with experimental data of the spectrum; the second, more theoretical, was the plurality of theories that the blackbody research required to compare and made consistent (electromagnetism, thermodynamics, and mechanics); the third, more epistemological, was a fascination with the universality of the blackbody spectrum that attracted those physicists like Planck who were in search for laws that could express "something of absolute" [4]:

"I had always looked upon the search for the absolute as the noblest and most worthwhile task of science" [37] (p. 46).

Planck assumed Wien's law (1896) as a fundamental reference in his studies_- "it must be necessarily true" [15] — for different reasons: it was consistent with the StefanBoltzmann equation (2), as well as with Wien's displacement law $\left(T \lambda_{\max }=\right.$ cost $)(1893)$; furthermore it fitted rather well with the experimental data until the experiments of Lummer and Pringsheim (about September 1900), which showed some experimental limitations at long wavelengths (see Fig. 4). The need to improve Wien's law became even more imperative in late 1900 in Berlin, when Planck was informed that Kurlbaum and Rubens, who were working on the energy of blackbody radiation with materials like fluorite, rocksalt, and quartz, had obtained further data that confirmed Lummer's and Pringsheim's results and hence partially disconfirmed Wien's law: the function did not fit the experimental data for high wavelengths of the radiation emitted by a blackbody [38]. The aforementioned situation is the incipit of Planck's paper:

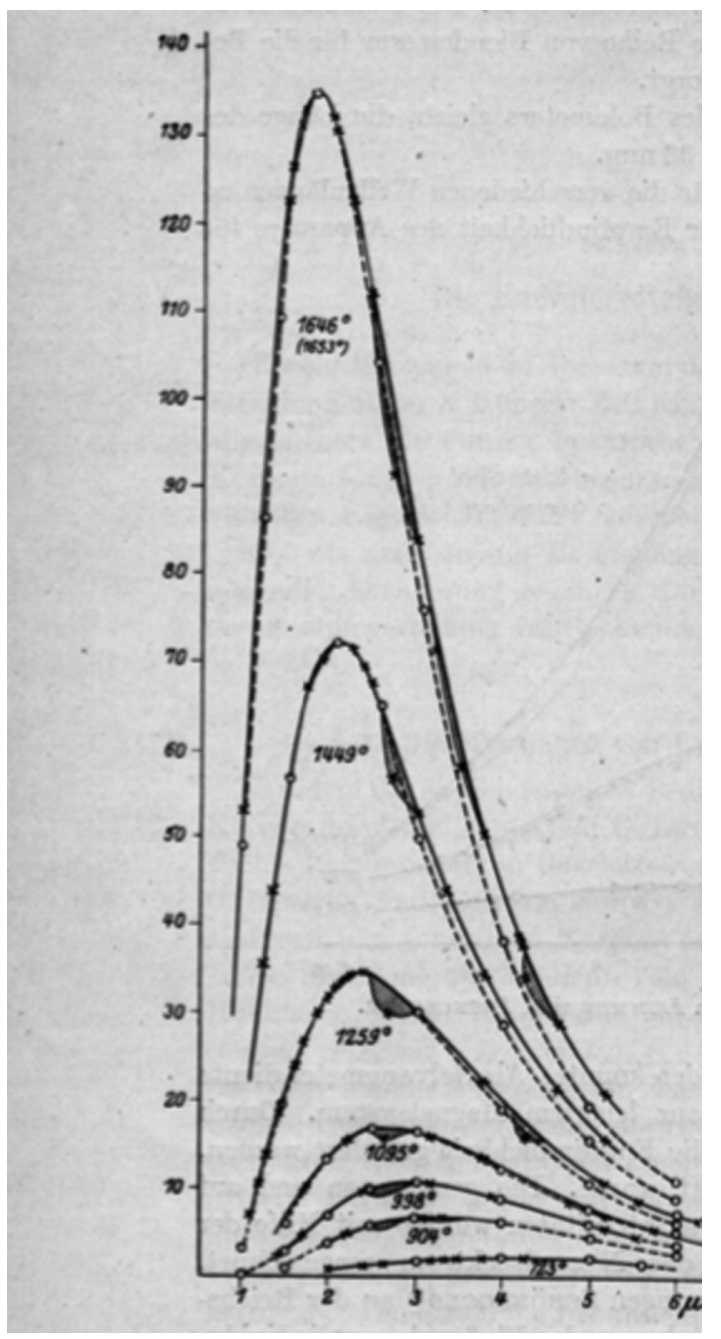

FIG. 4. Measurements carried out in 1900 at PhysikalischTechnische Reichsansalt (PTR) in Berlin Charlottenburg showing significant deviations from Wien's law (broken lines). The image is taken from Ref. [38] (p. 65).

"The interesting result of long wavelength spectral energy measurements which were communicated by Mr. Kurlbaum at today's meeting, and which were obtained by him and Mr. Rubens, confirm the statement by Mr. Lummer and Mr. Pringsheim, which was based on their observations that Wien's energy distribution law is not as generally valid, as many supposed up to now, but that this law at most has the character of a limiting case, the simple form of which was due only to a restriction to short wavelengths and low temperatures. Since I myself even in this Society have expressed the opinion that Wien's law must be necessarily true, I may perhaps be permitted to explain briefly the relationship between the electromagnetic theory developed by me and the experimental data" [15] (p. 1).

Even before the problematic experimental evidences, Planck was reasoning about the physical meaning of 
Wien's distribution law and he had already translated the law (1) in terms of

$$
u(\nu, T)=\frac{8 \pi \nu^{2}}{c^{3}} U(\nu, T),
$$

where $U(\nu, T)$ is the average energy of the resonators of frequency $\nu$ within the cavity and the coefficient represents the density of number of the oscillators with a frequency comprised between $\nu$ and $\nu+d \nu$ (the number of oscillators in an unitary volume). Simply by comparing the expressions (1) and (3), Wien's equation appears to be consistent with the hypothesis that the average energy of the oscillators with frequency $\nu$,

$$
U(\nu, T)=a \nu e^{-b \nu / T} .
$$

The exponential form of Eq. (4) drew its strength from its well fitting with experimental data, as well as its consistency with the Stefan-Boltzmann equation and with Wien's displacement law [39]. Neither a satisfying theoretical explanation nor a convincing model of resonators could support or justify it.

The crucial aspect of Planck's expression of Wien's equation, (3) and (4), is its effectiveness to focus on the processes of "energy exchange between resonator and radiation" [15] and reshape the problem so as to avoid, at the beginning, any hypothesis about the nature of the resonators. The idea was to find an improved formal expression of how energy of the single oscillator depends on $\nu$ and $T$ [an improved expression of $U(\nu, T)$ ], and, thanks to that, to achieve an improved version of the spectral energy. How can such a new expression be derived? How does it look?

We are now at the essential point of our reconstruction of Planck's reasoning: instead of following his peers who were debating on the mechanical or electromagnetic models of resonators and on the direct expression between the energy of the resonators and the temperature of the blackbody, Planck changed perspective and decided to address the problem from a pure thermodynamic point of view. The electromagnetic and mechanical routes explored so far appeared too ineffective to grasp the core of the problem: a new perspective was needed. As Planck retrospectively described:

"[the] analyses still showed even more clearly that an important connecting element or term, essential for the complete grasp of the core of the problem, must be missing. So there was nothing left for me but to tackle the problem from the opposite side, that of thermodynamics, in which field I felt, moreover, more confident" [40].
More specifically, Planck made the fundamental move to focus his attention completely on the concept of entropy and to search, in the relation between the entropy and the energy of the resonators, the route for a new expression of the average energy of the single oscillator. In his words:

"In fact my earlier studies of the Second Law of Heat Theory stood me in good stead, so that from the start I tried to get a connection, not between the temperature but rather the entropy of the resonator and its energy, and in fact, not its entropy exactly but the second derivative with respect to the energy since this has a direct physical meaning for the irreversibility of the energy exchange between resonator and radiation" [40].

\section{M-I-T analysis of "On an Improvement of Wien's Equation for the Spectrum"}

The reasoning developed by Planck in the first paper can be reconstructed in 8 steps, each of which can be identified as related to mathematization (M), interpretation (I) or technical aspects $(\mathrm{T})$.

(i) As first step, consistently with the choice of skipping the direct search for the expression of $U(\nu, T)$, Planck ignored the relation between the energy and the temperature that emerged from the theorem of equipartition of the energy $(E=k T)$ and that, instead, was taken into account by Rayleigh and Jeans. The entropic route led him to reformulate the problem as follows: assuming that $U(\nu)$, the average energy for the oscillators with a given frequency $\nu$, has to be related with $S(\nu)$, the entropy of the system of oscillators with frequency $\nu$, what relation between $S$ and $U$ can be found and how? (M)

(ii) As Planck wrote "Since I was, however, at that time still too far oriented towards the phenomenological aspect to come to closer quarters with the connection between entropy and probability, I saw myself, at first, relying solely upon the existing results of experience" [40]. Thus, as the second step, he found a way to highlight the relation between $U$ and $S$ : considering again Wien's expression of the average energy of the oscillators and reshaping it as a differential expression between energy and entropy. (M)

(iii) Concretely, the relation he found by analyzing Wien's equation is as follows:

$$
\frac{d^{2} S}{d U^{2}}=\frac{\alpha}{U}
$$

Thanks to this approach, the problem of finding $U(\nu)$ is turned into the technical problem to solve a differential equation and, hence, to find $U(\nu)$ moving backward with integral methods.

Indeed, if one considers the thermodynamics law (valid at constant volume), 


$$
\frac{d S}{d U}=\frac{1}{T}
$$

it is possible to re-obtain Wien's expression of $U(\nu, T)$ in Eq. (4) and, then, Wien's expression for $u(\nu, T)(1)$. The consistency between Eqs. (4) and (5) encouraged him to proceed in his reasoning since Wien's law was working so well with a large range of $\lambda=c / \nu$ to represent a robust and reliable starting point that only needed to be slightly modified to cohere with new empirical data. (T)

(iv) At this point, Planck goes on and modifies (5) making conjectures about its form: "Following this suggestion I have finally started to construct completely arbitrary expressions for the entropy which although they are more complicated than Wien's expression still seem to satisfy just as completely all requirements of the thermodynamic and electromagnetic theory." [15] (italic added). (M)

(v) In order to formalize this constraint, Planck added a generic term $\beta$, proportional to the frequency $\nu$ :

$$
\frac{d^{2} S}{d U^{2}}=\frac{\alpha}{U(\beta+U)}
$$

Citing Planck [15]: "It [Eq. (7)] is by far simplest of all expressions which lead to $S$ as a logarithmic function of $U$-which is suggested from probability considerations-and which moreover reduces to Wien's expression for small values of $U . "$ (T)

(vi) Integrating the differential equation (7), with respect to $U$, using Eq. (6) and the calculus method to integrate rational functions, the following expression of $1 / T$ can be derived:

$$
\frac{1}{T}=\frac{\alpha}{\beta} \log \frac{U}{\beta+U}
$$

Finding $U$ in the equation (using exponential functions and arithmetical properties), he obtained this expression of $U(T)$ : (T)

$$
U(T)=\frac{\beta}{e^{-\beta / \alpha T}-1} .
$$

(vii) Placing the expression of $U(T)$ in $u(\nu, T)$, he came to a generic expression depending on $\beta$ and obtained the general form of his law: (T)

$$
u(\nu, T)=\frac{8 \pi \nu^{2}}{c^{3}} U(\nu, T)=\frac{8 \pi \nu^{2}}{c^{3}} \frac{\beta}{e^{-\beta / \alpha T}-1} .
$$

(viii) The last step consists in posing the expression (8) of $1 / T$ equal to the one derived by Wien's law (5). This step allowed one to obtain:

$$
\frac{\alpha}{\beta} \log \frac{U}{\beta+U}=\frac{1}{c_{2}} \log \frac{U}{c_{1}} .
$$

The comparison between the two expressions leads to the following relations between the constants:

$$
\begin{aligned}
& \frac{\alpha}{\beta}=\frac{1}{c_{2}}=-\frac{1}{b \nu} \\
& \beta+U=c_{1}=a \nu
\end{aligned} \Rightarrow \begin{aligned}
& \alpha=-\frac{\beta}{b \nu} \\
& \beta \sim \beta+U=a \nu
\end{aligned} \Rightarrow \begin{aligned}
& \alpha=-\frac{a}{b} \\
& \beta=a \nu
\end{aligned} .
$$

Thanks to the previous passages he arrived at the final expression that represents Planck's improvement of Wien's distribution (1): (T)

$$
u(\nu, T)=\frac{8 \pi \nu^{2}}{c^{3}} \frac{a \nu}{e^{b \nu / T}-1} .
$$

The values of the constants $a$ and $b$ that Planck could obtain and calculate are [4] $a=0.4818 \times 10^{-10} \mathrm{~s}{ }^{\circ} \mathrm{C}$, $b=6.885 \times 10^{-27} \mathrm{erg} \mathrm{s}$. " 'The universal constant' $b$ had the same value as the more famous universal constant $h$ in 1900 , while the universal constant $a$ was to become, again in 1900, part of the constant $k(k=b / a) "$ [4].

\section{Comments on the M-I-T analysis of the first phase}

If we focus our attention on the M-I-T analysis, we can see that most steps in phase 1 are technical and the other steps regard mathematization processes. From the point of view of mathematical thinking [41], the steps (iv)(v) include a conjecture about the form of the analytical expression of $d^{2} S / d U^{2}$.

This conjecture led Planck to find an expression of the spectral density that was more consistent with empirical data but did not suggest any physical interpretation of the phenomenon happening within the cavity. This was the main problem he addressed in the following weeks: to find another way to re-obtain the expressions of the average energy of the single oscillator with frequency $\nu$ (9) and, hence, of the distribution law (13). This new approach allowed their meaning to be physically interpreted. As we will see next, in order to reach this outcome, Planck still focused his attention on entropy but, this way, he considered Boltzmann's probabilistic approach.

\section{B. Phase 2: Construction of a model in analogy with Boltzmann's approach}

\section{The two months between Planck's first and second paper on the blackbody radiation}

The way that Planck faced the issues raised by his fruitful proposal for the expression of spectral density was explained by himself in his Nobel lecture [40]: "[...]even if the radiation formula should prove itself to be absolutely accurate, it would still only have, within the significance of a happily chosen interpolation formula, a strictly limited 
value. For this reason, I busied myself, from then on, that is, from the day of its establishment, with the task of elucidating a true physical character for the formula, and this problem led me automatically to a consideration of the connection between entropy and probability, that is, Boltzmann's trend of ideas; until after some weeks of the most strenuous work of my life, light came into the darkness, and a new undreamed-of perspective opened up before me."

In 1877, Boltzmann had introduced the concepts of microstates (or complexions) and macrostates to interpret entropy and the issue of irreversibility in the second law of thermodynamics. His task was not simply to deduce the energy distribution at equilibrium, but to show the entropic relation between this distribution and that of an arbitrary state, so as to explain why a system evolves naturally toward the equilibrium state. Boltzmann's crucial step was to ascribe a probability to each macrostate in terms of the number of complexions corresponding to it [42]. By maximizing the state probability so defined, Boltzmann had shown that the equilibrium state, i.e., the state with the maximum value of entropy, is characterized as being the state with a probability higher than any other possible distribution. Reasoning this way, he had obtained

$$
W=\frac{(N+P-1) !}{(N-1) ! P !},
$$

which expresses the number of complexions corresponding to the distribution of $P$ packages of energy over $N$ molecules. This relation and the approach are borrowed by Planck and lie behind the reasoning that led him to write the December 14 paper. The paper does not explicitly state the reasoning and it also contains several ambiguities that Planck himself tried to fix in follow-up papers [16,43] and that historians of physics have analyzed in depth $[9,44,45]$.

As Planck wrote, "Elsewhere I will soon give a detailed account of the considerations I have only hinted at here, as well as a retrospective look at the development of the theory so far" [8].

\section{M-I-T analysis of "On the Theory of the Energy Distribution Law of the Normal Spectrum"}

For our purposes, in following Planck's reasoning, we will focus only on the main passages that led him to interpret the formal expression he presented in the October paper. These passages have the overarching goal of searching for a way to provide a physical meaning to the entropy of the resonators of the cavity and, in the wake of Boltzmann, to find a way to count the complexions corresponding to a macrostate characterized by an average energy $U$. In more detail:

(i) As a starting point, Planck stressed his aim to reobtain the expression (13) from physical assumptions and, in particular, from a physical model of the interaction between radiation and matter in the cavity. (I)

(ii) Through a formal analogy with a similar problem (Boltzmann's equilibrium in gases), he conjectured that the distribution of energy over the resonators might follow a similar rule. In particular, Planck considered the cavity at thermal equilibrium and split the total energy into two marcoparts: the energy of the radiation field and the energy $E_{0}$ distributed over the resonators in the cavity. Next, he focused on $E_{0}$ and imagined to have $E_{0}=E+E^{\prime}+E^{\prime \prime}+\cdots$ where $E$ is the energy distributed over the $N$ resonators of the cavity that have frequency $\nu, E^{\prime}$ is the energy distributed over the $N^{\prime}$ resonators with frequency $\nu^{\prime}$ and so on. Then, he considered "the distribution of the energy over the separate resonators of each group, first of all the distribution of the energy $E$ over the $N$ resonators of frequency $\nu^{\prime \prime}$ [8]. (M)

(iii) After the first step, he was ready to introduce the passage that makes this paper a milestone in the birth of quantum physics: "If $E$ is considered to be continuously divisible quantity, this distribution is possible in infinitely many ways. We consider, however-this is the most essential point of the whole calculation $-E$ to be composed of a very definite number of equal parts and use thereto the constant of nature $h=6.55 \times 10^{27} \mathrm{erg}$ s. This constant multiplied by the common frequency $\nu$ of the resonators gives us the energy element $\epsilon$ in erg, and dividing $E$ by $\epsilon$ we get the number $P$ of energy elements which must be divided over the $N$ resonators. If the ratio is not an integer, we take for $P$ an integer in the neighborhood" [8]. (M)

(iv) Thanks to the previous guess, Planck was able to find a way to count the finite number of complexions corresponding to the equilibrium state of a system on $N$ resonators with frequency $\nu$, over which $P$ units of energy (each equal to $h \nu$ ) are distributed. The number of complexions $W$ is expressed by the relation (14) (note that the process of counting includes ambiguities that were discussed and fixed, starting from the works of Ehrenfest and Einstein, see Refs. [9,46] for a detailed discussion of this issue). ( $\mathrm{T}$ )

(v) The same calculation can be made for each group of oscillators and the multiplication of all these numbers gives $R_{0}$ : "all possible complexions for the arbitrary assigned energy distribution over all resonators" [8]. (T)

(vi) Given the expression of $R_{0}$, Planck could express the entropy of the system of resonators as $S=k \log \left(R_{0}\right)$, the expression of Boltzmann entropy that nowadays looks familiar to us, thanks to the introduction of the constant $k$. To pursue the goal to re-obtain $U(\nu, T)$ 
and, hence, $u(\nu, T)$ the last move was to go back to energy and to a relation that could depend on temperature: "After the stationary energy distribution is thus determined using a constant $h$, we can find the corresponding temperature $\theta$ in degree absolute using a second constant of nature $k=1.346 \times$ $10^{-16} \mathrm{erg} \mathrm{deg}^{-1}$ through

$$
\frac{1}{\theta}=k \frac{d \log \left(R_{0}\right)}{d E_{0}} .
$$

The product $k \log \left(R_{0}\right)$ is the entropy of the system of resonators; it is the sum of the entropy of all separate resonators" [8]. (M)

(vii) The December paper proceeds with the following statement: "It would, to be sure, be very complicated to perform explicitly the above mentioned calculations, although it would not be without some interest to test the truth of the attainable degree of approximation in a simple case. A more general calculation which is performed very simply, using the above prescriptions shows much more directly that the normal energy distribution determined in this way for a medium containing radiation is given by

$$
u_{\nu} d \nu=\frac{8 \pi \nu^{3}}{c^{3}} \frac{d \nu}{e^{h \nu / k \theta}-1} . "
$$

Planck closes the paper by stressing the importance of the calculation in deriving a precise value to the $k$ constant. ( $\mathrm{T}$ )

\section{M-I-T analysis of "On the Law of the Energy Distribution in the Normal Spectrum"}

The reasoning presented in the December talk was explained in the 1901 paper, where Planck also provided tips for the calculation of the new constants. In the following, the M-I-T analysis of this paper shows how the reasoning is merely technical and deductive for calculation (T). Every step is to be considered implicitly marked with $\mathrm{T}$ :

(i) Once this assumption was made and expressed $S$ in terms of the logarithm of $W$, Planck had to derive his function $u(\nu, T)$. Since $W$ was expressed by means of factorials depending just on $N$ and $P$, and since $N$ 's and $P$ 's are big, he used Stirling's approximation for the factorial:

$$
\log (x !)=x \log (x)-x .
$$

$U$ and $\epsilon$ could be reintroduced by using $U=\epsilon P / N$ so $P / N=U / \epsilon$. At this point it was possible to go back to $\nu, T$. For this purpose, he expressed the entropy of one oscillator of the group $G(\nu)$ expressing $S$ of the group in terms of $U$ and $\epsilon$ and dividing by $N$ :

$$
S=k\left[\log \left(1+\frac{U}{\epsilon}\right)+\frac{U}{\epsilon} \log \left(1+\frac{\epsilon}{U}\right)\right] .
$$

The value of $\epsilon$ is not expressed analytically in terms of $\nu$, but its existence is hypothesized a priori according to mathematical conjectures.

(ii) Recalling now a general form of the solution of (7),

$$
\frac{d S}{d U}=\frac{\alpha}{\beta}[\log (U)-\log (\beta+U)],
$$

and expressing the constants $\alpha$ and $\beta$ in terms of $k$ and $h$, he obtained the following:

$$
\frac{d S}{d U}=-\frac{k}{h \nu}[\log (U)-\log (h \nu+U)] .
$$

Deriving $S$ in Eq. (18),

$$
\frac{d S}{d U}=-\frac{k}{\epsilon}[\log (U)-\log (\epsilon+U)],
$$

the expression (20) becomes equal to the expression (21) if $\epsilon=h \nu$.

\section{Comments on the M-I-T analysis of the second phase}

The second phase is articulated in two moments, the process starts with mathematization moves and then goes on through technical operations, even if the statement of the problem concerns the interpretation of the result obtained technically in the first phase (step 1). Indeed, Planck had approached the problem from the thermodynamic perspective, but he had no models for the entropy in this specific case and he was obliged to carry out an analogy with another problem, using formal expressions that had been elaborated by Boltzmann in the case of gases.

A critical and conceptual point of the first logical step was to interpret the meaning of entropy - and of "disorder," as Planck said - of a resonator, which has some specific features that make it partially different from the meaning of entropy that Boltzmann attached to gas. As Badino stresses,

"In the former case [the case of resonators], the disorder takes the form of "natural radiation," a particular assumption on the incoherent variation of the Fourier components of the waves exciting a resonator. [...] in a gas the disorder is a characteristic of a set of molecules at a given instant, while in cavity radiation, it is a characteristic of the temporal evolution of individual resonators. It is precisely this shift of meaning that allows Planck to introduce the entropy for a single resonator, a concept that would not make sense in gas theory" [9] (p. 15).

This point is expressed by Planck in different papers as follows: 
"Entropy means disorder, and I thought that one should find this disorder in the irregularity with which even in a completely stationary radiation field the vibrations of the resonator change their amplitude and phase, as long as one considers time intervals long compared to the period of one vibration, but short compared to the duration of a measurement." [8] (p. 1).

"In the thermal oscillations of a resonator the disorder is temporal, while in the molecular motion of a gas it is spatial. However, this difference is not so important for the calculation of the entropy of a resonator as it might appear at first sight; because through a simple consideration can be stressed what is essential for a uniform treatment" [47].

Yet, the most critical issue that Planck had to address is precisely that which led him to formulate the discretization hypothesis (step 3): to find a theoretical limitation to the possibility of distributing energy among the groups of resonators and, then, to avoid the inconsistency between the data and the model that would have been implied by an infinite entropy. This necessity obliged Planck to turn once again to formal conjectures to be interpreted a posteriori, rather than moving forward from the problem to the model and from the model to the technical operations. He mapped meanings by means of an analogy between different domains and addressed the critical issues that emerged in the comparison: he formulated a mathematical conjecture- the value of an element of energy must be fixed for every frequency, namely, the discretization hypothesis - to keep the consistency between the constraints that limited the target domain (interaction radiation-matter) and the computation procedure for complexions imported by the source domain (gas theory).

The process of interpretation required thus a new conjecture based on mathematical arguments to make sense physically of Planck's law (16) and derive it theoretically within a new theory, after having conjectured its form only by means of a creative act. The physical interpretation of this hypothesis is one of the most interesting and discussed topics in the history of physics (see Refs. [3,9]).

In the third paper, the derivation is presented organically and the new steps are presented in a deductive (technical) manner; this technical procedure, which aims at comparing the empirical and theoretical laws, leads to the values of the constants $h$ and $k$ but also to the relation between energy and frequency that was destined to become so important in quantum physics.

At this stage, two crucial interpretative issues emerged: (i) to interpret the assumption that $E$ exchanged between resonators and radiation must be divided in equal packets for every group of resonators with the same frequency; (ii) to interpret the proportionality between the energy of a packet and the frequency, $\epsilon=h \nu$, that emerged formally equating the expressions for the first derivative of $S$ Eqs. (20) and (21).

\section{Discussion of the overall M-I-T analysis}

The MIT analysis carried out on Planck's original papers shows to what extent blackbody is a rich case to investigate the interplay between mathematics and physics. It allowed us to see, in tangible form, when mathematics played a instrumental or technical role for physics and when it played a structural role. As for this second case, the analysis made emerge, in Planck's reasoning, both examples of mathematization processes (mapping meaning to mathematics) and of interpretation (mapping mathematics to meaning) [1,23]. Furthermore, the analysis allowed us to recognize how mathematics worked as an instigator of scientific revolutions [22] and how mathematics was for Planck as source of creative power [30]: mathematics provided him with unique reasoning strategies, including formal analogies, which led him to formulate his distribution law and re-achieve it from an alternative view.

We cannot deny that the case of blackbody is complicated. Thanks to the analysis, at least two reasons for this implicit complexity can be pointed out. The first is that blackbody is a typical "border problem" [6] and the theater of the clash between different models, perspectives, languages: on the one hand, we have electromagnetism, with its mathematics of continuum and its imaginary based on fields and waves; on the other hand, we have thermodynamics, with its mathematics of discrete and its imaginary based on particles.

The second reason is that Planck's argumentation is a refined and very articulated construction where a special role is reserved to hypotheses, assumptions, formal conjectures, and formal derivations. They are all forms of advanced mathematical thinking that, in this case, show their potential in physics since they guided and supported a breakthrough that undermined the basis of theories. Thus, to follow Planck's reasoning it is necessary to grasp the epistemological status of elements of an argumentation that blends empirical and theoretical, mathematical and physical pieces of reasoning: in brief, Planck's reasoning is complicated since it reaches the core of what an authentic interdisciplinary perspective is and proves that such a perspective is necessary to grasp the mechanism of this breakthrough.

For all these reasons, we believe that this chapter of physics is a masterpiece of thinking and should be taught at the university level. However, we also think that this case can become prototypical of activities aimed to develop a critical and aware attitude toward interdisciplinarity.

In the next section we briefly describe how we turned this analysis into a tutorial and how students reacted to it.

\section{IMPLICATION FOR THE TEACHING: THE TUTORIAL AND COMMENTS FROM STUDENTS}

Relying on the previous analysis, we designed a prototypical activity that addresses the goal of encouraging 
university students' awareness of the existence and the structure of interdisciplinary reasonings in physics. The activity was expected to be not too general to obey only epistemological principles, but not so specific to be only tailored on this case. We did not propose thus only a general reflection, but an in-depth analysis of a specific disciplinary case that was used as a case study from which making more general discourses arise.

Our reflections resulted in a tutorial comprising of a text to be analyzed and sets of questions. In this section we describe the structure and general features of the tutorial that, in our opinion, make it a prototype that can be used as a reference to produce analogous documents on other physics topics, where the structural role of mathematics is crucial.

Then, we briefly describe a pilot study we carried out with university students and lay out some data we collected. Such a description will allow us to close the paper reporting students' reactions and, through them, start discussing the educational potential of the approach.

\section{A. Description of the tutorial}

The main document of the tutorial is a discursive text punctuated by mathematical tasks and empty boxes that students are asked to fill so as to "complete" the missing steps in the reasoning, applying technical mathematical procedures [1]. In accordance with the model of Udhen et al. [1], the second part of the tutorial asks the students to reconstruct, on their own but working in groups, some technical processes belonging to the external loop (c), which are framed in the general argumentative thread, showing explicitly (by means of suitable sentences) the places and roles of said processes in the dialectic alternance of mathematization and interpretation phases.

Not filling in the tasks does not compromise understanding of the main discourse, since the necessary information to go forward was reported after every task. They are useful in that they deepen the connections between different formulas, encouraging engagement with the specific mathematical strategy, stepping into scientists' shoes when faced with a choice or looking for an idea on how to proceed. However, students who do not succeed in finishing one task can still go on reading without missing information.

In writing the discursive text, we took care to underline only the crucial details needed to reproduce a faithful picture of the original process and its main articulations. We were careful not to befuddle the issue with too many details that could make this revolution even harder to grasp rather than clarifying it. We wrote the discursive part of the text of the tutorial with care, following linguistic strategies of text organization to guarantee textual cohesion [48], with the objective of making explicit the reasoning, conjectures, and argumentations, and the role that mathematics can play in the development of physical theories.
The tutorial was designed for a three-hour group activity, preceded by a one-hour historical frontal lesson about blackbody, from the description of Kirchhoff's cavity to the theoretical approach and the bodies' emission-absorption problem based on the "absolute" model of a blackbody, the resonators model, and the laws considered valid at that time.

The tutorial, reported in the Appendix, is divided into three main sections. First, the students are asked to read the text without filling in the "missing parts," and then to answer a first set of questions aimed at guiding them to encounter the topic and to identify the points that are crucial for their comprehension of Planck's reasoning and those that they understand less and on which they need further clarification. In the second part, the students, divided into groups, are asked to fill in the missing part of the text. The final part consists in a second questionnaire, which aims at making the students reflect on the interplay between mathematics and physics, both in this specific case and in general. To do so, an introduction to Udhen et al.'s model [1] is necessary. This questionnaire has a double goal: on one hand it asks the students to recognize and highlight in the text the aspects they retain technical and those they see as structural, reflecting how the different aspects affect their comprehension of Planck's reasoning. On the other hand, it sets out to encourage general reflections on the contribution of the tutorial to thinking critically about interdisciplinarity. Each student is invited to provide individual answers to the questions and to discuss their answers with the other members of the group.

The activities should lead to a collective discussion.

\section{B. Contexts and methods of the tutorial pilot implementation}

We experimented the whole activity (lesson, tutorial, and discussion) twice, in a course of physics education within the physics degree Master's at the University of Bologna (1st year), held by the same teacher, in two consecutive years (2016-2017 and 2017-2018) and the number of students involved was 58 in all (27 and 31). The two contexts were very similar, as well as the composition of the groups: each group had an approximately equal male to female ratio, the students had a background in physics (about $50 \%$ ) or mathematics (about $30 \%$ ) or astrophysics (about 20\%). No students belonging to minority cultural groups were present.

In both of the validation trials, the time devoted to the activity was, as planned, three hours. Regarding the tutorial, the first section took about 30 minutes. For the second section, we divided the class into groups of 4 to 5 students, mixing students with different backgrounds (physics, astrophysics, mathematics). The group activity took about 90 minutes (including breaks) and, during it, besides the teacher (O. L.), two instructors (L. B.; A. C.) interacted with the groups asking them to explain what they 
were discussing and what critical issues they were experiencing. The remaining time (half an hour), was devoted to the last section (individual answer to the questionnaire and group discussion). After the tutorial, the teacher mediated a classroom discussion, that lasted almost 30 minutes in both cases, to collect their reactions and eventually foster interactions and further discussion among the students. A fruitful variation in the second experimentation is that the discussion was semiguided: before collecting spontaneous students' comments, we proposed to them two topics for the discussion, recalling the final questionnaire: the role of mathematics and physics in this case and the aspects of the activities that impressed them more, starting from their written answers.

We collected the text filled in by the groups (part 2) and the individual written answers (parts 1 and 3) to the questionnaire in the Appendix. We audio recorded with tablets the group works and the final discussion and we transcribed all the audio recordings.

For the purpose of this paper, we carried out a phenomenological qualitative analysis aimed to flesh out the macro evidences about students' reactions to the activity, in order to check whether the tutorial has the potential to enlarge the students' span of the possible ways math and physics may interplay. In particular we went through the data in order to (i) point out students' difficulties in technical and conceptual aspects as they emerged from the written answers and the group work transcripts (parts 2 and 3 of the activity); (ii) check the effectiveness of the tutorial against students' reactions to the meaning of interdisciplinarity between mathematics and physics they grasped from the tutorial (parts 2 and 3); (iii) search for unexpected results emerging from students' answers in the questionnaire (part 3) and the discussion, to enlarge the list of possible impacts of the activities on the students beliefs about interdisciplinarity. The researchers (authors of the paper) who collected and analyzed the data were the teacher and the instructors themselves.

\section{Students' reactions}

In the following we report some reactions, stressing both what we expected or desired and some unexpected results, as well as the difficulties which emerged.

As we expected, students did not find the activity simple. Some difficulties regarded technical aspects: some students claimed to need more help in matching an analytic expression with physical constraints (task 2); others were not familiar with combinatorics (required in the last part of the tutorial, task 5).

Other difficulties mentioned by the students were due to the following:

- unknown terminology (e.g., spectral density, resonators, complexions) and lack of connections with their usual terminology;
- the need to know more technical information about the physical laws used as starting points of the tutorial, in order to accept them as such;

- epistemological issues, such as why scientists look for analytical expression of physical quantities and why it is important to stress whether a principle is used or ignored in a derivation (absolute conception of science).

In general, although not simple, the students did not perceive the tasks as too difficult or out of their reach: the problems were caused mainly by divergence from their normal habits.

In particular, the technical tasks, even when they were not completely solved, played a fundamental role in guiding the students to analyze the text and in grasping the salient elements of Planck's reasoning which led to the quantum scientific breakthrough. Moreover, they allowed the students to reflect deeply on the interdisciplinarity between mathematics and physics.

Here we report the main results of our implementation, which showed the effectiveness of our tutorial in partially reaching the goals we set out during the planning stages and which encouraged us to proceed with testing the didactic value of tutorials with this structure in real didactic contexts.

1. The students appreciated the reconstruction of historical sources since it helped them fill the gap left open by the usual presentation of the role of Planck in the birth of quantum physics.

Most of the students said that, thanks to this tutorial, they understood for the first time the real problem faced by Planck; they had the feeling of following his train of thought, his logical path, his choices. One student commented: "It doesn't go into excessive detail but still allowed me to follow his train of thoughts. The first conjecture was particularly important: mathematics is not very hard, but it allowed me to understand the sense of Planck's amendment to Wien's law."

In general, they found it engaging to have the possibility of dealing directly with a historical reasoning that triggered a scientific breakthrough.

2. The mathematical details necessary to grasp the nature and key points of the breakthrough were identified as crucial for their understanding by more than $80 \%$ of the students who showed a nontrivial conception of the interplay between mathematics and physics.

In their comments, most of the students recognized the passage to the entropic point of view and its role in the scientific breakthrough as, at the same time, new and crucial for their understanding of Planck's reasoning. Students who had seen this topic before had never focused on this change of variable of observation and its theoretical background, which is indeed key to Planck's proposal.

Most of the students who mention the two conjectures as crucial points for their understanding recognized also that this breakthrough is due to a special relationship between 
mathematics and physics: a mathematical conjecture allowed Planck to fit experimental data better than Wien, but this expression required physical interpretation and theoretical framing. A significant number of students said that it was the first time that they saw how "an advance in physical theory happens as justification of a purely mathematical procedure." The other side of the coin is constituted by those students - a very few-who did not focus on the crucial steps in the reasoning: they commented by reporting the most traditional instrumental-contextualization relationship and did not delve deeper into the innovation either on the disciplinary or on the epistemological or interdisciplinary side. As a general trend, we observed that a conception of the interplay between mathematics and physics wider than a trivial view (instrumental or application) was necessary for students to grasp Planck's reasoning.

3. The tutorial was considered well balanced in details and contextualization of the mathematical procedures by more than two-thirds of the students.

Several students said that the mathematical activities clarified some of their initial doubts. Most of the students highlighted as fundamental steps the ones we had introduced in the text to anticipate the desired or expected "finish lines" of the reasonings, or to come back to the whole reasoning, clarifying Planck's choices of assumptions, laws, variables, analogies, hypotheses, and his conjectures. In general, the students considered the sentences that made the technical and structural roles of mathematics explicit as relevant for their comprehension, in particular when they previewed the formal procedures. This is an example of students' comments of this kind: "The previews are crucial for the understanding of the whole text, as much so as a lot of links that I found in the text. They allowed me to prepare myself for the reading, to focus on the problem in question; the technical aspects allowed me to convince myself of the formulas and what had been anticipated."

4. Most of the students enlarged the span of the possible ways math and physics may interplay, recognizing in some cases the role of mathematics in triggering scientific breakthroughs.

The tutorial encouraged the students to develop profound reflections about the possible relationships between mathematics and physics, with a special attention to the role of mathematics as instigator of scientific revolutions [22] and mathematics as structural in physical modeling [1]. One significant example is the following, in which a student displayed evidence of having reflected deeply on the different possibilities and of moving carefully from one to the other, identifying the distinct character of each approach. Some students focus their comments on the specific case, while others take the opportunity to go beyond. Andrea belongs to the first group, while Silvia and Paolo to the second.
Andrea: "This tutorial highlights the strong connection between mathematics and physics and the fact that this connection goes far beyond a mutual dependence between two separated disciplines: mathematics is not only useful to 'make the calculations' in physics and physics is not only a mere application of mathematics 'soiled' by reality. They are conflated and the one opens new horizons for the other, brings to it new questions, and, consequently, implies a progress. Planck's deductive process showed us that science would be very limited if it were compartmentalized. In the beginning, Planck found an answer to his questions in mathematics, then he found a physical interpretation that, maybe, would never have entered his mind, given the counterintuitive nature of the physical hypothesis he had to impose. In this case mathematics opened the door to the discovery of new physical concepts. This dynamic of interpenetration between these two disciplines is not usually shown, even though other examples could be found, like differential calculus, relativity."

Silvia: "Even though I had seen 'phenomena of interdisciplinarity,' I had never seen something so explicit, so it was very interesting."

Paolo: "Mathematics and physics seem to cooperate closely when scientists want to reach something new. Sometimes the step forward is due to a mathematical intuition, sometimes a physical one."

Andrea, like other classmates but in a clearer way, stressed that the instrumental or application mathematics-physics relationship would not have allowed an understanding of this breakthrough. It is the intertwining between the two (interdisciplinarity) that creates progress in the other, introducing new dynamics. He stressed that without mathematics Planck would not have reached the physical revolutionary idea because it is counterintuitive, so mathematics opened the route for new physical concepts. The last aspect grasped by the student is very refined: formal mathematical steps could open new paths, even revolutionary, since scientists could be blocked, rather than helped, by their intuition and strong beliefs about how things should work.

5. The structure of the tutorial encouraged promising metareflections about the way students deal with mathematics in physics learning, which could help them in the future in adopting a more sophisticated approach towards text reading and problem solving in physics.

In the final discussion of the last implementation, the students were asked to comment on the activity in terms of interdisciplinarity between mathematics and physics and its role in this scientific breakthrough. In some students' comments, encouraging traces are visible of the effectiveness of our tutorial in leading university students along the right track to becoming able to play, a little at a time, the two most intellectually complex epistemic games identified by Tuminaro and Redish [23]. 
Yet, the combination of the second phase-experience of use of mathematics in the reconstruction of a physical creative process - and the third phase - analysis of the most important steps for their comprehension of Planck's reasoning-triggered interesting reflections by the students about their needs related to the interplay between mathematics and physics for understanding physics in depth. Two different needs were stressed in particular by the students, which they realized to be important for their comprehension of physics; for each, we provide a brief description and a prototypical comment:

(a) To make sense physically of formal manipulation

"The text helped me to add more physical laws so it's not only mathematics, but you need the critical sense of a physicist to fix the constraints, choose the correct rule, because otherwise it seems that formulas are simply modified at random... why did he choose this variable, why this way?"

(b) To go into mathematical technical details

"The need for half and half [technical details and general description of the physical issue] was not immediately obvious to me because I thought that I didn't need to go into detail... why do I need mathematics if I have understood the problem and what is outlined, why do I need to go into the mathematical formalism to understand? But, actually, to understand in depth what I am talking about, I then realized I need to progress to 'numbers,' to the technical aspects."

These needs expressed by the students reveal that, when mathematics is involved in their ordinary physics learning activities, they have an unexpressed feeling of discomfort. Sometimes they feel the lack of a bigger picture that makes sense of technical choices; in this specific case, they refer to the meaning of formulas and the assumptions and motivations underlying a technical choice of variables. In other cases they try to understand avoiding the technical mathematical details, which they do not perceive as crucial, but, on the contrary, as avoidable appendices in a general physical discourse. These results concern physics teaching and learning activities in general and are interesting to us for at least two reasons. First, the tutorial activated in the students a process of metareflection about their approach to the study of a text in order to learn a new physical topic. Furthermore, in some cases the students reported attitudes towards the use of mathematics in physics teaching and learning that mirror two of the epistemic games presented by Tuminaro and Redish, and become aware of these during the discussion. In the first example, the students realize that they often perceive mathematical procedure in textbooks as void and casual; this belief resonates with the ones that guide the students when they play recursive plug and chug and the students identify the cause in the usual lack of information about physical reasoning that underlies the manipulation of formulas. In the second comment we recognize the student's tendency to search for a physical mechanism without moving to the mathematical formalization before the tutorial, and a change in her beliefs about the relevance of technical details in order to grasp physics in depth. Considering the results of Tuminaro and Redish [23], who stressed the trivial use of mathematics in physics by students in problem-solving activities and the inadequacy of traditional teaching in encouraging activation of the most sophisticated epistemic games, these metareflections are promising. Moreover, it seems that such metareflections were induced by the structure of the tutorial rather than by the specific content.

\section{CONCLUSION AND DISCUSSION}

Two research questions guided the study reported in this paper: What instructional materials and activities can support university teaching to highlight the structural role of mathematics in the development of new ideas in physics, and overcome the tendency to reduce it to a mere technical instrument [1,17-20]? What is the effect of these materials and activities on students' understanding, epistemologies and skill development? In this paper we described a study carried out to provide a possible answer to the first one and open a preliminary discussion on the second one.

The model of Udhen and colleagues [1] guided us to develop an interdisciplinary analysis of Planck's original papers thanks to which we could reconstruct his reasoning. The analysis showed that highlighting the structural and technical roles of mathematics in physics is necessary to grasp the nature of one of the most important and famous breakthroughs in the history of physics, as well as the beauty and richness of Planck's reasoning. On the basis of M-I-T analysis, we built up a tutorial intended for university students.

As we reported in Sec. II, other historical cases have similar features. This encourages us in hypothesizing that the general structure of our analysis and of the tutorial can be applied to create, mutatis mutandis, teaching materials with the same overarching goals but about other topics. So this tutorial can be seen as a kind of prototype.

The main features of the approach, which we consider essential in order to build similar teaching materials on different topics, are as follows:

(1) fleshing out from the original papers examples of mathematization and interpretation processes and making explicit the role of the technical steps;

(2) letting the students gain experience of, and reflect on, the specific local use of mathematics in some crucial phases of the whole argumentative thread, guided by specific tasks and explicit questions;

(3) guiding the student to reflect critically, both on their own and in groups, on the nuances of interdisciplinarity and their role in their learning process;

(4) guiding them to share their reflections in classroom discussion in which the teacher rephrases and scaffolds their experiences and intuitions. 
The previous four points move from the aim to provide analytic tools to recognize and unpack the interplay between mathematics and physics to the aim to develop metacognitive and epistemological competences.

To test the potentiality of the approach, we implemented the tutorial with two classes of university students, as a pilot study. Students' answers are promising and showed that such an approach made most students understand the real contribution of Planck's work on blackbody radiation to the birth of quantum physics. Even though some students had difficulties in dealing with a teaching proposal slightly different from their usual experience, most of them, interacting with the others and the tutors, grasped the nature of the breakthrough, the crucial steps of the reasoning and solved the mathematical tasks. Going beyond the specific topic, in most cases the students enlarged their span about possible interdisciplinary relationships between the two disciplines and showed awareness of the evolution of their conceptions due to our teaching activity. In their comments, some students made analogies and comparison with other physical topics they had studied, moving to more general comments about the role of mathematics in the understanding of physics.

Supported by such preliminary evidence, we are planning to investigate in the future whether and how the approach is effective to address the challenge posed by Tuminaro and Redish [23] and hence is able to affect university students' attitudes, epistemologies, and skills in dealing with the interplay between mathematics and physics and in problem solving activities. We have started refining the tutorial presented in this paper with the aim of scaffolding, in a more explicit way, students' reflections about the productive role of an interdisciplinary lens in their approaches to physics learning.

As a further development of the research, we are designing similar tutorials for high school students and in-service teachers, with suitable adaptations and simplifications (change of topic and difficulty of the tasks), in order to check whether and how our approach could contribute to foster a better integration between mathematics and physics in secondary teaching.

\section{ACKNOWLEDGMENTS}

A. C. is supported by the "National Group for Algebraic and Geometric Structures, and their Applications" (GNSAGA-INdAM).

\section{APPENDIX: TUTORIAL FOR UNIVERSITY STUDENTS}

\section{Questionary}

Part 1) Read by yourself the text, without filling in the required tasks, and answer the following questions.

(i) Did you understand or discover something new with respect to what you knew before? Highlight it in the text. (ii) What are the crucial points for your comprehension of Planck's reasoning? Highlight them in the text, writing comments in a paper sheet.

(iii) What are the steps that you understood less and you want to clarify? Why? Highlight them in the text, writing comments in a paper sheet.

Part 2) Carry out with your group the tasks proposed in the text, that allow you to "touch with your hands" the mathematical steps in Planck's reasoning.

Part 3) Answer by yourself the following questions and discuss the answer within your group.

(a) Are some mathematical steps relevant for your comprehension? Are them technical, structural or the both, referring to Udhen et al.'s model?

(b) Compare the steps you highlighted in Part 1 with the ones you mentioned answering question a. Are there common points? Did the activities help you to clarify some of your initial doubts?

(c) Did the mathematical activities change your vision of the interdisciplinarity between mathematics and physics? If so, how and why?

(d) Thinking about your reading and comprehension strategies of a scientific text before this tutorial, did you find something new in this text? Did the activities influence your approach?

\section{Text: "Blackbody and the hypothesis of discretization"}

\section{Hypothesis formulation}

At the turn of 1900, the attention of physicists all over Europe was focused on a fundamental problem: to determine a function describing the density of energy emitted by a blackbody with a constant temperature $T$ (thermodynamic equilibrium state). Planck reached the formulation of his expression for the density of energy following a procedure radically different from his contemporaries and succeeded in justifying it from a theoretical point of view. To do this, he had to introduce a revolutionary hypothesis: the discretization of the process of interaction between radiation and matter. This hypothesis was not accepted in the beginning; on the contrary, immediately it gave rise to many doubts in his own creator.

The first part of the text has two main goals: (i) to compare the peculiarities of the different approaches followed by the physicists who worked on the blackbody issue; (ii) to highlight the hypothetical-conjectural reasoning that led Planck, in 1900, to express his version of the function. The second part of the text focuses the attention on the arguments that brought Planck to interpret from a physical point of view the mathematical conjecture he introduced as an "act of desperation."

1- Wien's distribution

Before Planck, the attempts to construct a function describing the density of energy emitted by a blackbody at a constant temperature were mostly focused on finding 
an analytic expression for the density of energy. They all relied on thermodynamic arguments, empirical laws extrapolated from experimental data and mechanical and thermodynamic models of the energy of the oscillators of the Kirchhoff cavity.

Wien, using a general thermodynamic law, according to which the density of energy depended only on the product between $\nu^{3}$ and a generic function of $\nu / T$ and using mechanical and thermodynamic models of the energy of the oscillator inspired by Maxwell distribution, came, in 1896, to the following expression:

$$
u(\nu, T)=\frac{8 \pi \nu^{3}}{c^{3}} a e^{-b \nu / T},
$$

with $a, b$ positive constants.

From this expression it is possible to derive the more famous Wien's displacement law of 1893, according to which the wavelength $\lambda_{0}$, corresponding to the maximum of the emitted radiation energy, is inversely proportional to the body temperature.

Activity 1: Verify the previous statement, using the density function proposed in (A1). (Recall that the frequency and the wavelength are connected by the relation $\nu=c / \lambda$.)

On 1900 October 7, the German physicist Rubens met Planck in Berlin and showed him the results of the experiments he had carried out together with his colleague Kurlbaum on the radiation energy of the blackbody using material as fluorite, salt, and quartz. An unexpected fact arose: for long wavelength (order of magnitude tens of micron), Wien's distribution did not fit the experimental data. In the evening of the same day, Planck found the new equation that gave rise to the first quantization hypothesis. How?

\section{2- Planck's distribution}

Since the choice of focusing directly on the search for the density of energy function did not bring to a satisfactory solution of the problem, Planck decided to completely change perspective when tempting to modify as little as possible Wien's distribution law: he decided not to focus on the models of interaction between the radiation and a single oscillator in the cavity, but to consider the entropic properties of the system. Through these properties he analyzed and "modified" the Wien's distribution law.

Planck knew that to obtain an expression for the spectral density was enough to find an expression for $U(\nu, T)$, the average energy of a single oscillator with frequency $\nu$, since he proved that the following relation held between these two quantities:

$$
u(\nu, T)=\frac{8 \pi \nu^{2}}{c^{3}} U(\nu, T) .
$$

How to compute therefore the expression for $U$ ? Here comes the novelty. In 1900, it had been already known for 30 years that a harmonic oscillator in thermodynamic equilibrium state had, classically, the average energy
$U=k T$ : this is the equipartition theorem for energy. Probably Planck did not believe in the validity of the theorem and did not apply it: if he had done so, he would have not found his law. Planck, on the contrary, decided to focus on the entropy $S$ of a single oscillator and to look at it as a function of the energy $U$, in order to find a differential relation between $S$ and $U$ that might suggest some analytical features of the function without determining, at first, an analytic expression for it. Since Wien's distribution law was efficient in interpolating empirical data for most of the spectrum values, Planck decided to start from this equation when searching for the new relation between $S$ and $U$ and to modify it according to the new constraints.

Let us see some details of the process.

Evaluating, in two different ways, the infinitesimal increase of entropy of a system with several identical oscillators at the thermodynamic equilibrium, Planck obtained the following expression:

$$
\frac{d^{2} S}{d U^{2}}=\frac{\alpha}{U},
$$

with $\alpha$ constant. From this expression and using the relation

$$
\frac{d S}{d U}=\frac{1}{T},
$$

which is as an expression of the second principle of the thermodynamic, valid at the thermodynamic equilibrium and with constant volume of the cavity, Planck re-obtained Wien's distribution (A1).

IIf there is time, the group could try to prove the compatibility of (A1) and (A3), keeping in mind (A4) and (A2)].

If, on one hand, this fact convinced the German physicist of the, at least partial, validity of the new equation, on the other hand it forced him to face the same problem of experimental data misfit that had emerged in the case of Wien's law.

At this point Planck, following, as he wrote, mathematical neatness criteria, modified his version of the Wien's distribution law, expressed in the form of the differential equation (A3). More precisely, he introduced in the equation a parameter $\beta$, directly proportional to the frequency $\nu$, such that for high frequencies (i.e., for big values of $\beta$ ) the new equation tended to Wien's one, while for low frequencies (i.e., for small values of $\beta$ ) the entropy $S$ depended logarithmically on $U$, as suggested by the thermodynamic approach. Among the following laws
(A) $\frac{d^{2} S}{d U^{2}}=\frac{\alpha(\beta+U)}{U}$,
(C) $\frac{d^{2} S}{d U^{2}}=\frac{\alpha}{U \beta}$,
(B) $\frac{d^{2} S}{d U^{2}}=\frac{\alpha \beta}{U^{2}}$,
(D) $\frac{d^{2} S}{d U^{2}}=\frac{\alpha}{U(\beta+U)}$, 
all obtainable from Wien's law by means of easy changes, the one proposed by Planck is (D).

Activity 2: In the light of the previous considerations, explain why this equation is preferable to the others, in order to respect the constraints that the German physicist decided to assume.

Activity 3: Using (A4) and starting from the correct expression among the four proposed before, determine the value $U$ of the energy of a single oscillator.

The obtained formula depends on $\alpha$ and $\beta$. The parameter $\beta$, as previously said, is directly proportional to the frequency; in particular we will see in the following that $\beta=h \nu$, where $h$ is precisely Planck's constant. For $\alpha$, Planck will get $\alpha=-k$, as described in the second part of the text.

Activity 4: In the expression for $U$ obtained in the previous activity, replace $\alpha$ and $\beta$ with the values just established ( $-k$ and $h \nu$ respectively) and use (A2) to obtain the density of energy $u$ as in the new model developed by Planck.

It is interesting to notice that the two constants $h$ and $k$ appeared at this moment for the first time.

Indeed, also $k$ had never been explicated before: it was Planck who named it "Boltzmann's constant" in honor of Boltzmann, recognizing a link between his research and the studies of the Austrian physicist, that used $k$ without naming it explicitly. Boltzmann's studies were indeed fundamental for the physical interpretation of the density of energy distribution hypothesized by Planck.

3- The importance of "looking" at the right variables

The choice of Planck to focus on the relationship between the entropy and the energy of a single oscillator was crucial. Indeed, some years later, the physicists John Rayleigh and James Jeans assuming, as Planck, that the cavity was at the thermodynamic equilibrium but working directly on $u$ using the classical model of the energy of a single oscillator $(U=k T)$, obtained the following function:

$$
u(\nu, T)=\frac{8 \pi \nu^{2}}{c^{3}} k T
$$

that, for low frequencies, coincides with Planck's function.

This proposal has, however, as a consequence, the socalled ultraviolet catastrophe; it implies that total energy $E$ is infinite, since,

$$
\begin{aligned}
E & =\int_{0}^{+\infty} u(\nu, T) d \nu=\int_{0}^{+\infty} \frac{8 \pi \nu^{2}}{c^{3}} k T d \nu \\
& =\frac{8 \pi k T}{c^{3}} \int_{0}^{+\infty} \nu^{2} d \nu=\frac{8 \pi k T}{c^{3}} \lim _{\nu \rightarrow+\infty} \frac{\nu^{3}}{3}=+\infty
\end{aligned}
$$

Note that the same thing does not happen assuming Planck's distribution.
Interpreting the new relation: natura facit saltus

After introducing his distribution, through a mathematical conjecture that determines a little change of Wien's equation, Planck spent a week in the "desperate effort" to find a physical interpretation that could give theoretical plausibility to his new equation and make sense of the conjecture.

In order to do so, Planck focused on the entropy of the system and its relationship with the internal energy of the system of oscillators, modeled as a thermodynamic system. To construct his interpretative model, Planck particularly referred to Boltzmann's statistical approach and to the microscopic description of the system, neglecting the oscillators nature and focusing only on the ways in which the energy settled on the various oscillators. Let us see how.

4- The complexions

Let us suppose that the blackbody is made up of $N$ oscillators, called resonators, having frequency $\nu$ and energy $E, N^{\prime}$ having frequency $\nu$ and energy $E^{\prime}, N^{\prime \prime}$ having frequency $\nu^{\prime \prime}$ and energy $E^{\prime \prime}$, and so on. If the energy $E$ exchanged is a continuum quantity, it could be distributed over the resonators oscillating at frequency $\nu$, in infinite different ways. If, on the contrary, it consists of a very big number $P$ of "packages" of energy $\epsilon$-here is the revolutionary Planck's hypothesis-such that $P \epsilon=E$, then there is a finite number of possible distributions. An analogue consideration holds for $E^{\prime}, E^{\prime \prime}$, and so on. Planck assumed his hypothesis as true and went on computing the number of complexions, i.e., ways in which the packages could be distributed over resonators, under this "discretization hypothesis."

Activity 5: Show that the number of ways in which the $P$ packages could be distributed over $N$ resonators (complexions) is given by the formula

$$
W=\frac{(N+P-1) !}{(N-1) ! P !} .
$$

Remark: the resonators must be considered as distinguishable, while the packages indistinguishable. Moreover, consider the possibility of resonators having zero energy.

5- An example

Let us suppose that a system is made of 3 resonators with total energy $E=6 \epsilon$. There will be 7 different possible distributions (macrostates):

(A) 1 resonator having energy $6 \epsilon ; 2$ resonators having energy 0

(B) 1 resonator having energy $5 \epsilon$; 1 resonator having energy $\epsilon ; 1$ resonator having energy 0

(C) 1 resonator having energy $4 \epsilon ; 1$ resonator having energy $2 \epsilon ; 1$ resonator having energy 0

(D) 1 resonator having energy $4 \epsilon ; 2$ resonator having energy $\epsilon$

(E) 2 resonator having energy $3 \epsilon ; 1$ resonator having energy 0 
(F) 1 resonator having energy $3 \epsilon$; 1 resonator having energy $2 \epsilon ; 1$ resonator having energy $\epsilon$

(G) 3 resonator having energy $2 \epsilon$.

Activity 6: Consider each distribution (macrostate): in how many ways (complexions or microstates) can it be realized?

Activity 7: Denote with $N$ the total number of resonators and with $N_{j}$ the number of resonators having energy $j \epsilon$ with $j=0, \ldots, P$; which is the formula expressing the number of complexions corresponding to each macrostate?

Activity 8: Verify that the total number $W$ of microstates (summing over all possible macrostates), computed in activity 6, coincide with the value obtained using (A5) and replacing the unknowns with the appropriate values.

6- From complexions to the density of energy

After modeling the interaction matter radiation, Planck came back to the density function obtained via its initial conjecture in order to derive it theoretically within the model. To do this, he used Boltzmann's formula $S=$ $k \log \left(R_{0}\right)$, replacing $R_{0}$ with the product of the previously obtained expression for $W$ for resonators at frequency $\nu$ and analogue expressions for the groups of oscillators with other frequencies. Then he approximated the logarithms of factorials using the Stirling's formula $\log (x !)=x \log (x)-$ $x$, because of big $N$ 's and $P$ 's, and divided by $N$ 's. He obtain the following expression for the entropy of the single oscillator as a function of $U$ and $\epsilon$ :

$$
S=k\left[\log \left(1+\frac{U}{\epsilon}\right)+\frac{U}{\epsilon} \log \left(1+\frac{\epsilon}{U}\right)\right] .
$$

Using (A4) and (A2), he determined the analytic expression of $u$ as a function of $\epsilon$. The last thing to do was, at this point, establish the value of $\epsilon$. In order to do this, Planck imposed that the obtained formula has to fit with the empirical thermodynamic law, used also by Wien, that states that the spectral density depends only on the product between $\nu^{3}$ and a generic function of $\nu / T$. In this way he got $\epsilon=h \nu$ with the new universal constant $h$ (not depending on $\nu$ ) named, hereafter, Planck's constant.
[1] O. Uhden, R. Karam, M. Pietrocola, and G. Pospiech, Modelling mathematical reasoning in physics education, Sci. Educ. Netherlands 21, 485 (2012).

[2] J. Fischer and J. Ullrich, The new system of units, Nat. Phys. 12, 4 (2016).

[3] J. Büttner, O. Darrigol, D. Hoffmann, J. Renn, and M. Schemmel, Revisiting the Quantum Discontinuity (Max Planck Institute for the History of Science, Berlin, 2000), Vol. 150.

[4] N. Robotti and M. Badino, Max Planck and the 'Constants of Nature', Ann. Sci. 58, 137 (2001).

[5] M. Planck, Physikalische Abhandlungen und Vortrage (Vieweg \& Sohn, Braunschweig, 1958).

[6] C. Tarsitani, Il dilemma onda-corpuscolo (Loescher, Torino, 1983).

[7] R. Thom, in Le Labyrinthe du Continu, edited by J.-M. Salanskis and H. Sinaceur (Springer-France, Paris, 1992), p. 137.

[8] M. Planck, Zur Theorie des Gesetzes der Energieverteilung im Normalspektrum, Verh. Dtsch. Phys. Ges. 2, 237 (1900); [The Old Quantum Theory (Pergamon Press, Oxford, 1967)].

[9] M. Badino, The odd couple: Boltzmann, Planck and the application of statistics to physics (1900-1913), Ann. Phy. (Leipzig) 18, 81 (2009).

[10] A. Drago, in Atti del XXV Congresso Nazionale di Storia della Fisica e dell'Astronomia (2005), http://www.brera .unimi.it/sisfa/atti/atti2005/C08-Drago.pdf.

[11] R.C. Dougal, The presentation of the Planck radiation formula (tutorial), Phys. Educ. 11, 438 (1976).

[12] https://www.slac.stanford.edu/pubs/beamline/30/2/30-2carson.pdf.
[13] J. S. Walker, Physics (Addison-Wesley, Boston, 2009).

[14] D. Halliday, K. S. Krane, and R. Resnick, Physics (John Wiley \& Sons, Inc., New York City, 1992).

[15] M. Planck, Ueber eine Verbesserung der Wien'schen Spectralgleichung, Verh. Dtsch. Phys. Ges. 2, 202 (1900); [The Old Quantum Theory (Pergamon Press, Oxford, 1967)].

[16] M. Planck, On the law of the energy distribution in the normal spectrum, Ann. Phy. (Leipzig) 4, 553 (1901).

[17] R. Karam, Framing the structural role of mathematics in physics lectures: A case study on electromagnetism, Phys. Rev. ST Phys. Educ. Res. 10, 010119 (2014).

[18] R. Karam, Introduction of the thematic issue on the interplay of physics and mathematics, Sci. Educ. Netherlands 24, 487 (2015).

[19] S. Kapon and A. Merzel, Content-specific pedagogical knowledge, practices, and beliefs underlying the design of physics lessons: A case study, Phys. Rev. Phys. Educ. Res. 15, 010125 (2019).

[20] S. Kapon and M. Schvartzer, Nurturing sensemaking of, through, and with a mathematical model, in Proceedings of the Physics Education Research Conference 2018 (American Association of Physics Teachers, Maryland, 2018).

[21] C. Tzanakis, in Proceedings of History and Pedagogy of Mathematics, Satellite of ICME 2016 (IREM de Montpellier, Montpellier, France, 2016).

[22] S. G. Brush, Mathematics as an instigator of scientific revolutions, Sci. Educ. Netherlands 24, 495 (2015).

[23] J. Tuminaro and E. F. Redish, Elements of a cognitive model of physics problem solving: Epistemic games, Phys. Rev. ST Phys. Educ. Res. 3, 020101 (2007). 
[24] A. Collins and W. Ferguson, Epistemic forms and epistemic games: Structures and strategies to guide inquiry, Educ. Psychol. 28, 25 (1993).

[25] E. F. Redish, Research on Physics Education, Course CLVI, Proceedings of the International School of Physics "E. Fermi", edited by E. F. Redish and M. Vincentini (IOS Press, Amsterdam, 2003).

[26] A. diSessa, Toward an epistemology of physics, Cognit. Instr. 10, 105 (1993).

[27] W. Blum and D. Leiß, Modellieren im Unterricht mit der “Tanken”-Aufgabe. Gefälligkeitsübersetzung: Mathematical model building with the "refuelling"-problem, Mathematik lehren 128, 18 (2005).

[28] M. Pietrocola, in Connecting Research in Physics Education with Teacher Education, edited by M. Vicentini and E. Sasso (International Commission on Physics Education, 2008), Vol. 2, Sec. A2, http://www.iupap-icpe.org/publications/ teach1/ConnectingResInPhysEducWithTeacherEduc_Vol_1 .pdf.

[29] G. Pospiech in Proceedings of the 2006 GIREP Conference: Modelling in Physics and Physics Education, edited by E. van der Berg, T. Ellermeijer and O. Slooten (University of Amsterdam, 2008) p. 587.

[30] H. Kragh, Mathematics and physics: The idea of a preestablished harmony, Sci. Educ. Netherlands 24, 515 (2015).

[31] R. Frodeman, J. Thompson, and C. Mitcham, The Oxford Handbook of Interdisciplinarity (Oxford University Press, New York, 1990).

[32] M. Planck, Planck's Original Papers in Quantum Physics, edited by H. Kangro (Taylor \& Francis Ltd., London, 1972).

[33] G. Kirchhoff, Über die Verhältnis zwischen dem Emissionsvermögen und dem Absorp- tionsvermögen der Körper für Wärme und Licht, Ann. Phy. (Leipzig) 109, 275 (1834); [I. On the relation between the radiating and absorbing powers of different bodies for light and heat, London, Edinburgh, and Dublin Philos. Mag. J. Sci. 20, 1 (1860)].

[34] G. Kirchhoff, Abhandlungen über Emission und Absorption (W. Engelmann, Leipzig, 1898).
[35] https://phys.libretexts.org/Bookshelves/University_Physics/ Book\%3A_University_Physics_(OpenStax)/Map\%3A_ University_Physics_III_-_Optics_and_Modern_Physics_ (OpenStax)/6\%3A_Photons_and_Matter_Waves/6.1\%3A_ Blackbody_Radiation.

[36] https://en.wikipedia.org/wiki/Wien\%27s_displacement_law.

[37] M. Planck, Scientific Autobiographies and Other Papers (Citadel Press, New York, 1948).

[38] D. Hoffmann, in Revisiting the Quantum Discontinuity (Max Planck Institute for the History of Science, Berlin, 2000), Vol. 150, pp. 45-68.

[39] M. Born, Atomic Physics (Blackie \& Son, LondonGlasgow, 1969).

[40] M. Planck, The Genesis and Present State of Development of the Quantum Theory, Nobel Lecture (1920), https:// www.nobelprize.org/prizes/physics/1918/planck/lecture/.

[41] D. O. Tall, Advanced Mathematical Thinking (Kluwer, Dordrecht, 1991).

[42] L. Boltzmann, Über die Beziehung zwischen dem zweiten Hauptsatze der mechanischen Wärmetheorie und der Wahrscheinlichkeitsrechnung respective den Sätzen über das Wärmegle-ichgewicht, Sitzungsberichte der Akademie der Wissenschaften zu Wien 76, 373 (1877).

[43] M. Planck, Über die Natur des weissen Lichtes, Ann. Phys. (Leipzig) 4, 818 (1902).

[44] M. Klein, Max Planck and the beginnings of the quantum theory, Arch. Hist. Exact Sci. 1, 459 (1961).

[45] T. S. Kuhn, Black body Theory and the Quantum Discontinuity 1894-1912 (Oxford University Press, New York, 1978).

[46] M. Badino, The Bumpy Road: Max Planck from Radiation Theory to the Quantum (1896-1906) (Springer, New York, 2015).

[47] M. Planck, Vorlesungen über die Theorie der Wärmestrahlung (J. A. Barth, Leipzig, 1913); [The Theory of Heat Radiation (Blakiston's Son \& Co, Philadelphia, 1914)].

[48] A. Siddharthan, Syntactic simplification and text cohesion, Res. Language Computation 4, 77 (2006). 